\title{
iEat: An Interactive Table for Restaurant Customers' Experience Enhancement
}

\author{
George Margetis ${ }^{1}$, Dimitris Grammenos ${ }^{1}$, Xenophon Zabulis ${ }^{1}$, \\ and Constantine Stephanidis ${ }^{1,2}$ \\ ${ }^{1}$ Foundation for Research and Technology - HELLAS, \\ Institute for Computer Science (FORTH - ICS) \\ ${ }^{2}$ Computer Science Department, University of Crete \\ \{gmarget, gramenos, zabulis, cs\} @ics.forth.gr
}

\begin{abstract}
An active field of research today is the technological enrichment of everyday activities using augmented reality and ambient intelligence technologies. To this end, augmenting dinner tables is a challenging task, requiring a high-quality user experience unobtrusively supporting and enhancing the user's main goal: eating and socializing. This work presents an augmented restaurant table, facilitating customers' ordering and enhancing their experience through entertainment and socialization features, as well as through interaction with physical objects placed upon the table surface.
\end{abstract}

Keywords: augmented reality, ambient intelligence, interactive tabletop.

\section{Introduction}

The emergence of augmented reality and ambient intelligence has motivated several research efforts to pursue the technological enrichment of everyday life activities, mainly in the areas of home, workplace, health and education. Lately, however, the areas of socialization, communication and entertainment have gained increased interest for multimodal and ubiquitous interaction towards enhancing common activities such as dining.

To this end, initiatives that aim to entertain and foster human communication and socialization through dining have already appeared as commercial solutions. For instance, the Inamo and Touche Diner restaurants [1,2] try to provide technology enhanced entertainment and socializing during dinner.

This work presents an innovative smart restaurant table, named iEat that aims to enhance restaurant customers' experience in terms of entertainment, socialization, food selection and ordering, providing the users with natural interaction with the table itself, as well as with physical objects placed upon its surface.

\section{$2 \quad$ Related Work}

The concept of augmenting dinner tables is challenging, mainly due to the fact that the interactive features of the table should not overwhelm the user's main goal, eating 
and socializing with other diners. Nevertheless, the idea of providing new types of interaction and facilities for this everyday human habit has led to numerous approaches. For example, Dinner Party [3] provides entertaining interaction between a person dining on a table and objects placed on top of it. The user can move these objects casting virtual shadows on the tabletop while animated creatures which were initially hiding in these shadows eventually appear. Another example is Neon Racer [4], a multi-user augmented reality racing game that can be played on a tabletop setting, providing social interaction in open environments. Food Media [5] aims to engage people dining at different locations into social communication and entertainment, supporting an integrated teleconference system, movement of partner's dishes remotely, display of various information, graphics or slow-rate animations through controlled color changing on the tablecloth and transmission of remotely designed patterns or messages using real edible food. A similar approach presented in [6] aims to support remote guests in experiencing a sense of togetherness, playfulness and sharing in a dinner party.

The work presented here discusses an augmented restaurant table facilitating the dinner process and fostering socialization and communication of the diners. It provides alternative types of natural interaction through the use of infrared lightpens based on the work presented in [7], as well as through the positioning and movement of existing plates on the table surface, supporting thus a large degree of interaction freedom to the users.

\section{The iEat Table}

An experimental installation of the iEat system is illustrated in Fig. 1 (left) comprising a white surfaced restaurant table for two persons $(90 \mathrm{~cm} \mathrm{x} 80 \mathrm{~cm})$, an Intel Core 2 Duo PC, a HD ready projector lying above the table, as well as a high definition infrared camera and a Microsoft Kinect sensor, both placed adjacent to the projector overlooking the table surface. The high definition camera tracks light sources in the infrared spectrum as in [7]. The Kinect is employed in tracking plates
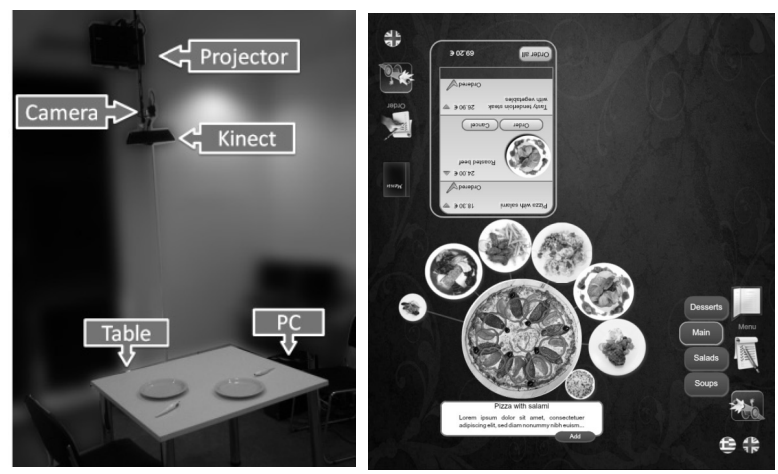

Fig. 1. Left: Experimental installation of the iEat table. Right: UI layout. 
on the table, which is achieved by analyzing the depth image and detecting plates through their circular height patterns.

Currently, user interaction is supported through moving / placing the plates on the table as well as through infrared lightpens, which are stylus-shaped, LED-based, infrared light (IR) emitters. The infrared lightpens emit light only when they touch the table surface, providing the following interaction gestures: (a) Click: Touch the table surface and instantly raise lightpen (abstaining from any lateral motion); (b) Down: Touch the table surface; (c) Up: Raise the lightpen above the table surface; (d) Move: Move the lightpen while touching the table surface.

\subsection{User Interaction}

The main objective of the iEat table is to augment restaurant customers' experience beyond standard activities performed at restaurants (i.e., reading the menu, ordering, chatting and eating) towards new levels of entertainment and communication. To this end, the iEat table provides a diversity of technology augmented facilities such as ordering, playing, decorating the table surface and communicating.
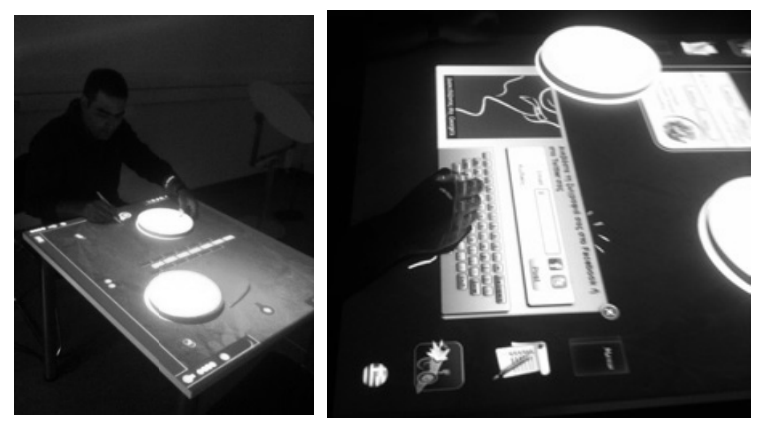

Fig. 2. Left: Playing games using plates and lightpen. Right: posting doodles and sketches to Facebook and Twitter.

\section{Selecting Dishes and Ordering}

The iEat system integrates an intuitive ordering facility enabling customers not only to find and order restaurant dishes, but also to get a preview of the dish that will be served. Fig. 1 (right) depicts the iEat ordering User Interface, which mainly consists of three components: (a) basic menu categories, (b) list of dishes and dish details for the selected category and (c) order list. The menu options and order list are placed at the customer's right hand side, while the dish details are projected upon and laterally an empty plate near the customer.

In more details, customers can explore the restaurant menu, by clicking on the main navigation menu button and then selecting one of the categories that have been expanded just next to it. By selecting a category, the iEat table displays thumbnails of the selected category's dishes around the empty dish which is nearest to the customer. If a customer clicks on a thumbnail, a larger photo is displayed on the surface of the 
dish, accompanied by additional details regarding for example its ingredients, nutrition facts, etc. The option for ordering the plate is also readily available for selection.

Furthermore, a customer is able to review the dishes in the order list and place the order. The order list summarizes all the dishes that have been added by the two diners, providing facilities for removing a dish from the list, view more details for a particular dish, order a specific dish and order all the dishes in the list. The iEat system provides an API for intercommunicating with restaurant ordering software, allowing its trouble-free installation and interoperation with systems already used in restaurants.

\section{Playing}

Aiming to entertain the diners while waiting for the dishes to be served, the iEat table features interactive games played using the empty plates and the lightpens. In the current prototype, a breakout type game has been developed (Fig. 2, left). The goal of each player is to hit as many tiles as possible, using the cannonballs launched by a cannon lying in front of the player. Moreover, players get more points if they manage to place their cannonballs to a particular opponent's territory. The aiming direction of the cannon is determined by the players moving their lightpens to the left or to the right in front of their cannons. Cannonballs can be repelled using plates as shields (in order to screen the opponent's cannonballs). Forthcoming versions of the iEat table will support additional games, accommodating a variety of potential player preferences.

\section{Decorating and Communicating}

Additionally, the iEat table supports facilities for decorating the table surface, through a library of alternative virtual backgrounds (tablecloths), as well as highly customizable drawing toolset (allowing users for example to select brush size and color, undo and redo action, etc.). Drawing and doodling is facilitated through the use of lightpens. Furthermore, users are able to post their drawings to their Facebook and Twitter accounts, sharing thus their experience with friends and followers (Fig. 2 right).

\section{Formative Evaluation}

The current iEat table prototype has been evaluated by four usability experts following the heuristic evaluation method [8]. In summary, sixteen usability problems were found, most of which were rated as aesthetic or minor. Two problems that were rated as major were the following: (i) the user cannot easily find out about the drawing options menu, since it is not included in the main navigation menu and it is displayed only if the users perform a click to a non-interactive table area, and (ii) if the dishes list contains more than seven items, the leftmost and rightmost dishes are displayed smaller in order to indicate that by selecting them the dishes carousel will 
rotate, however this will be difficult for users to find out. Furthermore, the evaluation also resulted in a list of the most interesting and usable system features, including among others the plates' interaction facilities, the overall look and feel of the application, as well as its sociability features.

\section{$5 \quad$ Conclusions and Future Work}

This work has presented an interactive restaurant table aiming not only to support ordering at the restaurant, but also to enhance customers' socializing and entertainment. The table features innovative interaction facilities, using the empty plates placed on the table and lightpens. The initial prototype of the iEat table has been evaluated by usability experts, having identified the strengths and weaknesses of the current implementation. Future work will address the usability problems that were found and will enrich the existing content, by adding beverages' information and additional entertainment games. Furthermore, user-based tests will be carried out aiming to assess diners' overall user experience.

Acknowlegdments. This work is supported by the FORTH-ICS internal 'Ambient Intelligence' RTD Programme.

\section{References}

1. Inamo restaurant, Soho, London - interactive oriental fusion restaurant and bar, http: / / www. inamo-restaurant.com/

2. Touche - Touch Table Diner Bar, http: / / www . touchediner.com/

3. Nam, H.Y., DiSalvo, C., Do, E.Y.L., Mendenhall, S.: Dinner Party sociable interfaces in a tabletop art project. In: First International Conference on Intelligent Interactive Technologies and Multimedia, pp. 306-310. ACM (2010)

4. Litzlbauer, W., Stuppacher, I., Waldner, M., Weilguny, M.: Neon Racer: Augmented Gaming. In: 10th Central European Seminar on Computer Graphics, CESCG (2006)

5. Wei, J., Wang, X., Tache, R., Peiris, R.L., Choi, Y., Halupka, V., Cheok, A.D.: Food Media: exploring interactive entertainment over telepresent dinner. In: 8th International Conference on Advances in Computer Entertainment Technology, p. 26. ACM (2011)

6. Barden, P., Comber, R., Green, D., Jackson, D., Ladha, C., Bartindale, T., Olivier, P.: Telematic dinner party: designing for togetherness through play and performance. In: Designing Interactive Systems Conference, pp. 38-47. ACM (2012)

7. Margetis, G., Ntelidakis, A., Zabulis, X., Ntoa, S., Koutlemanis, P., Stephanidis, C.: Augmenting physical books towards education enhancement. In: Workshop on UserCentred Computer Vision, in Conjunction with Workshop on the Applications of Computer Vision 2013, Florida (2013)

8. Nielsen, J., Molich, R.: Heuristic evaluation of user interfaces. In: SIGCHI Conference on Human Factors in Computing Systems: Empowering People, pp. 249-256. ACM (1990) 